\title{
Searches For Primeval Galaxies
}

\author{
C.A. Collins
}

October 28, 2018

\begin{abstract}
A primeval galaxy represents the earliest stages of a galaxy's life and as such provides clues to the early history of the Universe and the evolution of stars and galaxies. Over the last 20 years astronomers have been engaged in the quest to detect the faint signals from these objects, believed to lie at a distance comparable with the size of the Universe. A wide variety of observational techniques have been employed in this search, with astronomers eagerly awaiting each new generation of astronomical telescope or detector in the hope of finally solving the mystery to the origin of galaxies - or at least placing new and interesting constraints. Until recently, primeval galaxies have eluded detection in these searches, however experiments over the last couple of years which use either 10m-class optical telescopes or state-of-the-art submillimetre array detectors, may hold the clue to the origin of structure as they have finally uncovered what appears to be a widespread population of young galaxies.
\end{abstract}

\section{Introduction}

Primeval galaxies (hereafter PGs) can loosely be described as the progenitors of present-day galaxies, such as our own Milky Way, in the process of assembling their mass through gravitational collapse and forming their first generation of stars. Detecting the starlight from galaxies in such an early stage of their evolution has become one of cosmology's most elusive holy grails. The reason for this interest is that information about the ancestral characteristics of both spiral and elliptical galaxies helps observational cosmologists in their quest to map out the star formation history of the Universe, which, in turn, provides important constraints on galaxy formation theories.

There are already excellent reviews of galaxy formation, galaxy evolution and PG Searches in the astronomical literature: Koo and Kron (1992), Pritchet (1994) - on which section 4 of this article is based, Sandage (1995) - who provides an excellent historical account and Ellis (1997). The first part of this paper outlines the cosmological framework (section 2) and the physical processes underlying the growth of structure in the early Universe (section 3). The expected properties of PGs are described in section 4 and a summary of the previous observational quests is then given (section 5). Recent insights into star-forming galaxies from deep optical

\footnotetext{
${ }^{1}$ Author's address: Astrophysics Research Institute, School of Engineering, Liverpool John Moores University, Byrom Street, Liverpool L3 3AF, UK.
} 
surveys are discussed in section 6 and the possibility of dusty PGs is reviewed as a solution to their non-detection at other wavelengths (section 7 ). The paper concludes with a discussion of the likely productive avenues of the future (section 8 ).

\section{Cosmological Distance and Time}

Searches for PGs are really attempts to detect the furthest recognisable galaxies and thereby determine the epoch of galaxy formation. Let us begin by examining the relationship between the cosmological distance to a galaxy and the light-travel time over that distance; this will illustrate the general power of PG searches to probe the early Universe. Astronomers measure the distance to a galaxy using the property known as the redshift $(z)$. This is the term given to the increase in wavelength of light as it propagates through space, caused by the expansion of the Universe. The size of the redshift is a measure of how fast distant objects are moving away from the Earth and for distances not large compared to the size of the Universe $(z<0.1)$, the redshift can be related to the distance $(D)$ by Hubble's law, $D \simeq c z / \mathrm{H}_{0}$, where $c$ is the speed of light, and $\mathrm{H}_{0}$ is Hubble's constant. At larger redshifts the look-back time to a galaxy starts to become significant compared to the age of the Universe and the dynamics of the expansion, determined by its energy density content, becomes progressively more important. The basic relationship between look-back time $\Delta t$ from the present to a galaxy at redshift $z$ is given by

$$
\Delta t=\mathrm{H}_{0}^{-1} \int_{0}^{z}(1+z)^{-1}\left[(1+z)^{2}\left(1+\Omega_{\mathrm{M}} z\right)-z(2+z) \Omega_{\Lambda}\right]^{-1 / 2} d z,
$$

where $\Omega_{\mathrm{M}}$ and $\Omega_{\Lambda}$ are the contributions to the energy density from the matter and cosmological constant respectively. Figure 1 shows $\Delta t$ as a function of $z$ for three cosmological models which span the range of accecptable values for $\Omega_{\mathrm{M}}$ and $\Omega_{\Lambda}$. Despite the continuing debate in cosmology regarding the precise value of the quantities $\Omega_{\mathrm{M}}, \Omega_{\Lambda}$ and $\mathrm{H}_{0}$, observations of galaxies at $z \simeq 1-5$ always probe back to a time when the Universe was $\simeq 50 \%-10 \%$ of its current age respectively, in any plausible cosmology. This makes observations of galaxies at $z>1$ a very powerful tool for understanding the conditions in the early Universe and, not surprisingly, observations of distant objects, including searches for PGs, have over the years been extensive and wide-ranging: most, if not all, of the world's principal ground-based and space-born astronomical facilities, have, at some stage, devoted significant amounts of telescope time to primeval galaxy search programmes, covering the electromagnetic spectrum from the optical to the radio.

\section{Growth of Structure in the Big Bang Cosmology}

\subsection{The Cosmic Microwave Background}

The cosmic microwave background, discovered by Penzias and Wilson (1965), provides the strongest evidence for the hot origin of the Universe - otherwise known as the hot Big Bang. In this grand model, the radiation and baryonic matter were in thermodynamic equilibrium 


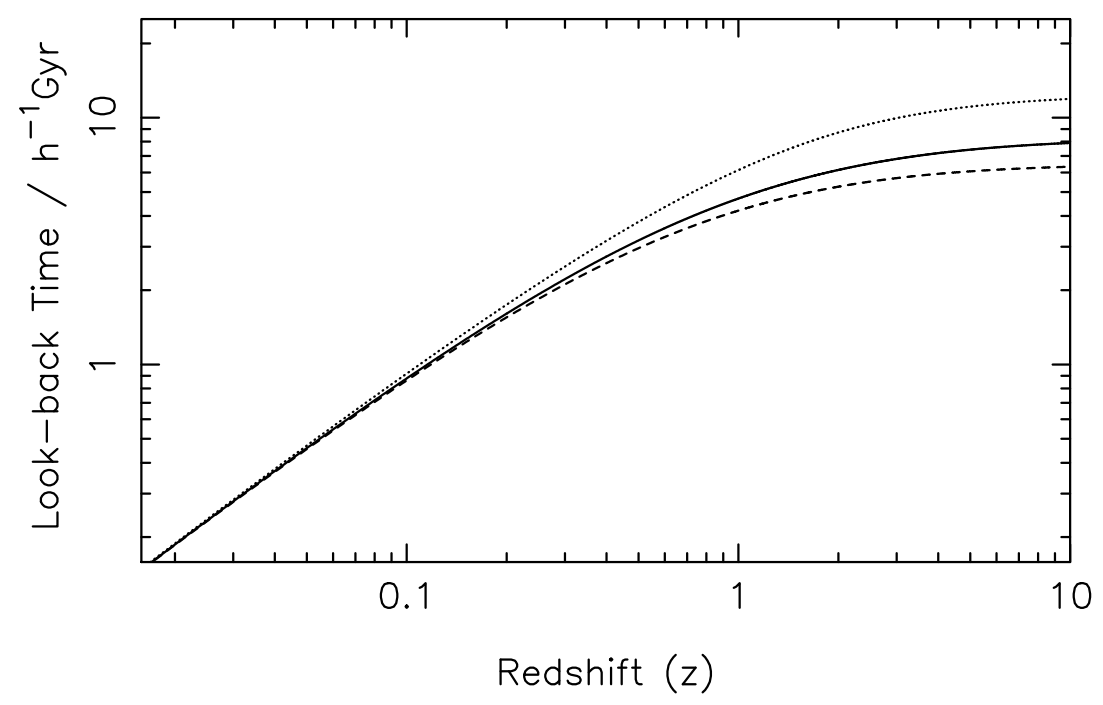

Figure 1: Look-back time as a function of redshift for the cosmological models: $\Omega_{\mathrm{M}}=1.0, \Omega_{\Lambda}=$ 0 (dashed line); $\Omega_{\mathrm{M}}=0.2, \Omega_{\Lambda}=0$ (solid line); $\Omega_{\mathrm{M}}=0.1, \Omega_{\Lambda}=0.9$ (dotted line). These models have been calculated for $\mathrm{H}_{0}=100 h \mathrm{~km} \mathrm{~s}^{-1} \mathrm{Mpc}^{-1}$, where $h$ expresses the uncertainty in the value of $\mathrm{H}_{0}$ taking values $h=0.5-1$ and $1 \mathrm{pc} \simeq 3.1 \times 10^{16} \mathrm{~m}$.

at sufficiently early times, which involved the constituent fundamental particles interacting with photons via Compton scattering. As the Universe expanded and photons redshifted so the temperature of the background radiation cooled. The radiation field last interacted with matter at a redshift $z \simeq 1000$, or some $10^{6}$ yrs after the Big Bang - at which time the temperature of the radiation had cooled to $\leq 3000^{\circ} \mathrm{K}$, enabling electrons to be captured forming neutral atoms.

It is believed that structures such as galaxies started out as small density fluctuations in this primordial soup of matter and radiation, growing by gravitational instability into larger overdensities as gravitationally bound systems were formed. The dense clumps of material in the gravitational potential wells caused collisional heating of the baryonic material, allowing rapid cooling of the gas by line radiation. Subsequently, larger clumps were formed as subclumps of material merged and combined - with the final state of the bound material governed by angular momentum conservation. The first stars formed in the dense cores, which enriched the primordial gas with heavy elements as a result of supernovae explosions.

In recent years there has been substantial empirical underpinning of this general picture of the early growth of structure. For example, the earliest density perturbations have now been detected as small temperature fluctuations $\left(\delta \mathrm{T} / \mathrm{T} \simeq 10^{-6}\right)$ in the cosmic microwave background by the Cosmic Microwave Background Explorer (COBE) satellite (see Smoot and Keay 1993). 
In addition, semi-analytical models of the growth of structure, which incorporate most of the known physical processes, have had success in reproducing many of the optical properties of galaxies in the most distant surveys (e.g. Baugh et al. 1998).

\subsection{Density Inhomogeneities in the Early Universe}

The density fluctuations inferred from COBE are remarkably small and we know that for the overdensities in our own galaxy $\delta \rho / \rho>1$; furthermore, gravitationally collapsed structures, such as quasi stellar objects, are known to exist at $z \simeq 5$, so by this time at least some perturbations must have grown past $\delta \rho / \rho=1$. The big questions for galaxy formation then are: What is the scale-size of the first collapsing overdensities? How fast can fluctuations grow in the early Universe? What sort of fluctuations must they be?

In 1902 James Jeans considered the criterion for gravitational collapse in an infinite uniform medium in which small density perturbations $(d \rho)$ give rise to adiabatic pressure changes or acoustic waves $(d p)$, such that

$$
d p=\mathrm{V}_{\mathrm{s}}^{2} d \rho
$$

where $\mathrm{V}_{\mathrm{s}}^{2}$ is the adiabatic sound speed. The criterion for collapse is satisfied by a mass (known as the Jeans Mass, $\mathrm{M}_{\mathrm{J}}$ ) just large enough that the sound crossing time is larger than the freefall collapse time - thus rendering the overdensity unable to respond with pressure changes fast enough to halt gravitational collapse.

It is straight forward to apply the basic idea of Jeans to the problem of galaxy formation in the early Universe and calculate the minimum spherical mass which would stop expanding with the universal Hubble flow, turn around, and gravitationally collapse. At $z=1000$, just after decoupling

$$
\mathrm{M}_{\mathrm{J}} \simeq 2 \times 10^{4}\left(\Omega_{0} h^{2}\right)^{-1 / 2} \mathrm{M}_{\odot}
$$

where $\Omega_{0}$ is the present mass density, and $\mathrm{M}$ is the unit of solar mass $\left(1 \mathrm{M}_{\odot}=2 \times 10^{30} \mathrm{~kg}\right)$.

This value assumes that the energy density of the Universe is matter dominated - at epochs earlier than about the decoupling stage $\left(z>1000\right.$ or $\left.t \leq 10^{6} \mathrm{yrs}\right)$, the energy density of the radiation field exceeds that of the matter content and the sound speed is then relativistic. During this period, known as the radiation dominated era, the minimum mass required to undergo gravitational collapse of baryonic overdensities rises to

$$
\mathrm{M}_{\mathrm{J}} \simeq 10^{14}\left(\Omega_{0} h^{2}\right)^{-2} \mathrm{M}_{\odot} .
$$

This mass is so large that it is in fact comparable to the entire baryonic content of each causally connected volume of space at any time during the radiation dominated era. Furthermore, the characteristic timescale required for matter fluctuations to collapse is longer than the characteristic time for the expansion of the Universe during this era. A combination of the large Jeans mass and rapid expansion time of the Universe guarantee that no baryonic fluctuations gravitationally collapse for the first $10^{6}$ yrs after the Big Bang and galaxy formation really only begins after this time at the onset of the matter dominated era. 
An estimate of the redshift at which galaxies collapse can be made using the Jeans criterion: an overdensity larger than $\mathrm{M}_{\mathrm{J}}$ will stop expanding and collapse in a time $t_{c}$, given by

$$
t_{c}=\left(\frac{3 \pi}{32 \mathrm{G} \rho}\right)^{1 / 2}
$$

Taking the example of our own Milky Way, the total mass implied from dynamical studies is $5 \times 10^{11} \mathrm{M}_{\odot}$ and from the distance of the furthest stars in the galactic halo a plausible size to the Galaxy before it collapsed is $\simeq 100 \mathrm{kpc}$. This implies a density $3.6 \times 10^{-24} \mathrm{~kg} \mathrm{~m}^{-3}$ and, using equation (5) a collapse time of $\simeq 10^{9}$ yrs, which corresponds to a look-back time of 7.2 Gyr if $\Omega_{\mathrm{M}}=0.2$ and $5.4 \mathrm{Gyr}$ if $\Omega_{\mathrm{M}}=1.0$ (all assuming $\Omega_{\Lambda}=0, h=1$ ). From figure 1 this translates to redshifts of formation $z_{f} \simeq 4-2$ respectively. If $h=0.5$ then formation redshifts range from $z_{f} \simeq 6-4$ for the same mass densities. Finally, bound objects the size of galaxies are unlikely to exist much above these redshifts for the simple reason that the age of the Universe is then short compared to the free-fall collapse time.

\subsection{Non-Baryonic Dark Matter}

It will not have escaped the attention of astute readers that the above physical arguments make no mention of the well known controversy regarding the possibility of missing mass in the Universe and the type of matter which might dominate the overall mass density. The most likely form of non-baryonic dark matter to dominate the mass density is known as cold dark matter, the primary candidates for which are in the form of Weakly Interacting Massive ParticleS or WIMPS - such as the super-symmetric lepton partners of bosons (e.g. Photino, Gravitino or Higgsino), which have predicted masses $\sim \mathrm{GeV}$. If the major constituent of $\Omega_{\mathrm{M}}$ in the Universe is in the form of cold dark matter then the growth of fluctuations begins before decoupling: the non-baryonic matter decouples from the radiation earlier and because it feels no radiation pressure can get on with the task of collapsing and forming gravitational potential wells during the radiation dominated era. At the onset of decoupling the baryonic gas, which until this time has been locked to the radiation field by electrostatic forces, falls very quickly into the gravitational potential wells created by the non-baryonic matter. In this case, assuming that the amplitude of density fluctuations decreases with scale, the first structures to form have a mass $\simeq 10^{5} \mathrm{M}_{\odot}-$ similar to the baryon-dominated case and again set by the pressure of the baryons after recombination.

To summarize, the variety of models represented in this short discussion of the growth of structure suggests that galaxies form by the collapse and subsequent merging of sub-galactic size units over a range of formation redshifts $\left(2 \leq z_{f} \leq 6\right)$. Uncovering PGs is one of the few ways of providing constraints on the various possibilities.

\section{Properties of PGs}

The two fundamental requirements of any PG search are a sound search strategy - covering a sufficient area of sky to a sufficient depth, and an ability to recognise a PG amongst the 


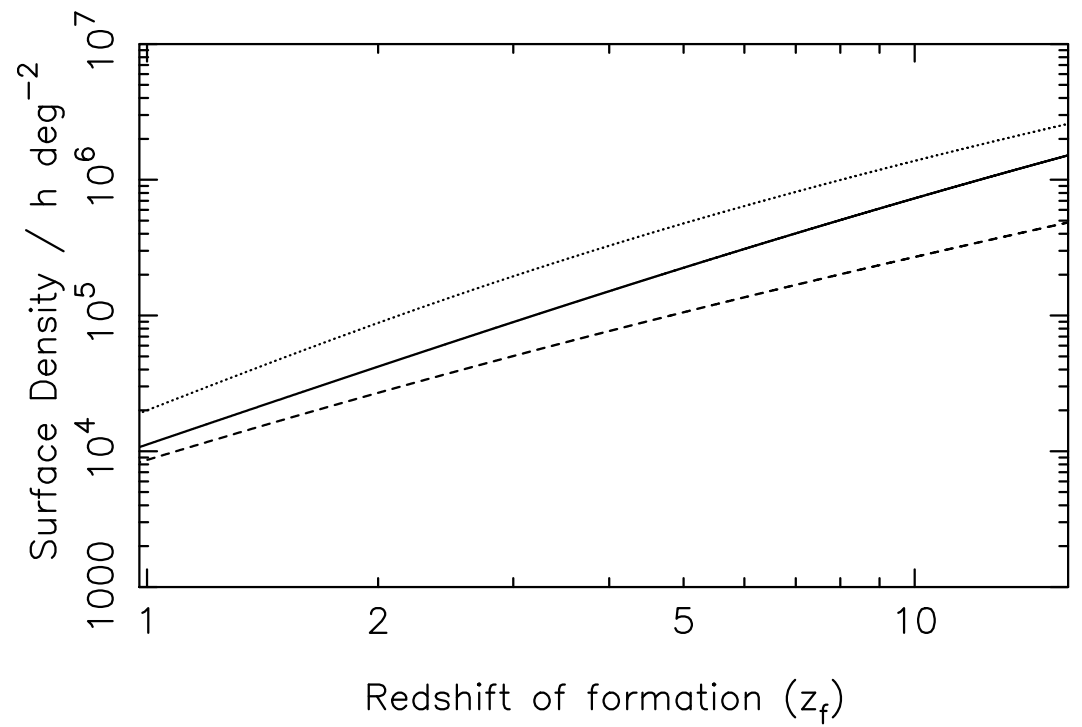

Figure 2: Expected surface density of PGs as a function of their formation redshift, assuming a collapse time of 1 Gyr. The three curves represent the same cosmologies as in Figure 1.

background of older and more normal galaxies. Therefore, it will be useful to have some idea of what the basic properties of such and object might be.

\subsection{Surface Density}

An estimate of the surface density of PGs on the sky can be made by extrapolating the volume density of local galaxies $\left(\sim 0.015 h^{3} \mathrm{Mpc}^{-3}\right)$ to high redshift. Suppose that all galaxies form at a particular redshift by gravitationally collapsing over a period of $1 \mathrm{Gyr}$, rapidly forming their first generation of stars: then the resulting areal density of PGs as a function of their formation redshift is shown in figure 2. PGs should be very abundant irrespective of the uncertainties in the cosmology, with at least $10^{3}$ in each area of sky the size of the moon!

\subsection{Angular Size}

This is one of the most difficult properties to estimate. The angular size of a PG depends upon when the bright phase occurred relative to the collapse as well as the redshift and cosmology. If the star formation occurs soon after the onset of collapse then PGs could be low-surface brightness objects with angular diameters 10-30 arcsec (Partridge and Peebles 1967). At $z \geq 5$ and with a surface density of $\geq 10^{5} \mathrm{deg}^{-2}$ (see figure 2) PGs would be virtually overlapping on 
the sky and very difficult to detect as discrete sources using conventional techniques. Possibly the best way to to detect such objects is to investigate the integrated background light resulting from such a population of sources and experiments to do just this have been carried out. At the other extreme is the idea, stretching back a number of years, that the star formation rate (hereafter SFR) is closely coupled to the gas density and hence the bulk of the star formation occurs in the central regions of collapsed PGs (Eggen, Lynden-Bell and Sandage 1962, Larson 1974). In this case we can expect the visible signature of PGs to have an angular diameter $\simeq 0.1-0.5$ arcsec, making them very difficult to resolve from the ground due to the limitation imposed by atmospheric "seeing" irregularities. Such objects would be excellent targets for optical satellite missions, such as the Hubble Space Telescope with its diffraction-limited 0.1 arcsec imaging capability.

\subsection{Luminosity}

The principal energy source of PGs is the release of binding energy in nuclear reactions at the centres of hot young stars. A crude estimate of the luminosity of a young galaxy can be obtained by simply considering the energy required to convert a fraction $Z$ of hydrogen into metals, based on the metalicities of old stars in the Milky Way, for which $Z \simeq 0.01$. A $\mathrm{PG}$ of mass $10^{11} \mathrm{M}_{\odot}$, with a star-forming phase lasting 1 Gyr (equivalent to a SFR of about $100 \mathrm{M}_{\odot} \mathrm{yr}^{-1}$ ) has a net bolometric luminosity $\simeq 6 \times 10^{37} \mathrm{~W}$. This makes PGs 10-100 times brighter than nearby bright spiral galaxies in which stars are being formed at a rate of a few $\mathrm{M}_{\odot} \mathrm{yr}^{-1}$. At $z=5$ this corresponds to an optical $(4500 \AA)$ flux of $10^{-18} \mathrm{Wm}^{-2}$ - at least 100 times fainter than the terrestrial night-sky background at these wavelengths; while in the near-infrared $(1-5 \mu \mathrm{m})$ the Earth's atmosphere is $\sim 1000$ times brighter than at optical wavelengths, making PGs at least $10^{5}$ fainter than the sky! For these reasons PGs are unlikely to be masquerading in existing catalogues of bright galaxies and can only hope to be detected by the largest telescopes.

\subsection{Spectral Energy Distribution}

In recent years significant progress has been made in reliably modelling the evolution of stellar populations in galaxies (e.g. Charlot et al. 1996). The basic approach, known as "isochrone synthesis", involves computing the evolutionary tracks of stellar populations for an instantaneous star burst (i.e. with no age dispersion) incorporating a finite rate of star formation. In this way the distribution of stars of various masses and ages can be modelled smoothly with time and isochrone synthesis models are found to reproduce well the observations of stellar populations in nearby galaxies if they formed more than a few Gyrs ago. This technique has been widely used to predict the major spectral characteristics of young galaxies $\leq 1$ Gyr after formation. Figure 3 shows the resultant spectral energy distributions for a galaxy forming stars at a rate of $1 \mathrm{M}_{\odot} \mathrm{yr}^{-1}$ observed after ellapsed periods ranging from $4 \times 10^{7} \mathrm{yrs}$ to $10^{10} \mathrm{yrs}$. The essential characteristic of figure 3 is that genuinely young galaxies radiate an almost constant energy density from $0.1-2.0 \mu \mathrm{m}$. The rise in the relative emission at longer wavelengths as the stellar population ages is due to the death of massive hot stars, whose lifetime $(t)$ varies with mass $(\mathrm{M})$ as $t \simeq\left(\mathrm{M} / \mathrm{M}_{\odot}\right)^{-2} \times 10^{10} \mathrm{yrs}$, which evolve into cooler red giant stars and supernovae. 


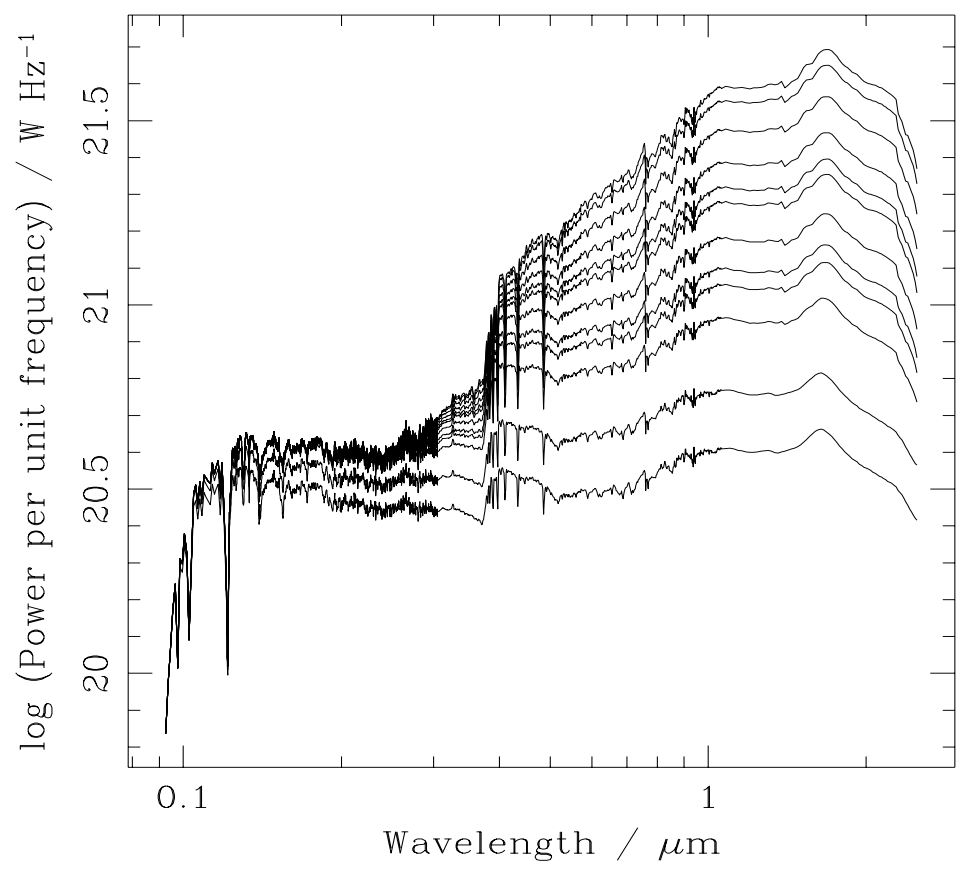

Figure 3: The spectral energy distribution expressed as power per unit frequency interval computed from stellar population synthesis code (see text) for a galaxy with mass $10^{11} \mathrm{M}_{\odot}$ and a constant SFR of $1 \mathrm{M}_{\odot} \mathrm{yr}^{-1}$ observed after a time (yrs): $4 \times 10^{7}$ (bottom curve), $10^{8}$, $3 \times 10^{8}, 5 \times 10^{8}, 6 \times 10^{8}, 10^{9}, 2 \times 10^{9}, 3 \times 10^{9}, 5 \times 10^{9}, 8 \times 10^{9}, 10^{10}$ (top curve). In these models Lyman $\alpha$ at $1215 \AA$ is shown in absorption. No extinction due to dust has been assumed (see figure 5)

One important consequence of the flat spectral energy distribution is that the measured optical or near-infrared flux of a PG is a direct measure of the instantaneous SFR within the system.

In addition to the stellar continuum emission, young star forming regions within the Milky Way and other nearby galaxies exhibit intense line radiation, principally the hydrogen recombination lines of Lyman $\alpha$ at $1215 \AA$ and the Balmer line $\mathrm{H} \alpha$ at $6562 \AA$. The Lyman $\alpha$ line is shown in absorption in figure 3 , but in star-forming regions these lines are seen in emission associated with the ionised hydrogen in the surrounding gas which constitutes the star-forming nebulae. The intensity of these lines is a measure of the ambient ionizing UV flux from young hot stars and can also be used as a tracer of star formation to estimate the SFR in an independent way from the spectral energy distribution (Kennicutt et al. 1987).

In conclusion, although predictions are sketchy, there are certain characteristics which PGs are likely to have that one can highlight: PGs should not be rare objects and can be identified in the optical or near-infrared by their flat continuum emission or intense line radiation. On the other hand, despite being intrinsically luminous objects, at least compared to normal galaxies, the likely formation redshift of PGs dictates that they are almost certainly faint and possibly of low-surface brightness, making them very difficult to detect. 


\section{Searches For PGs - An Historical Overview}

It would appear from the previous discussion concerning the likely properties of PGs that a search covering only a limited area of sky, but reaching to faint flux limits, most closely represents the optimum search strategy. The high number density of PGs expected also ensures that one part of the sky will be pretty much as good as any other in terms of the likelihood of detection - although clearly avoiding regions of high galactic extinction and high stellar density, such as the plane of the Milky Way or towards the Large Magellanic Clouds, is advisable. PG searches have been carried out for more than 20 years and have always used the largest, and therefore most sensitive, telescopes and accompanying instruments to conduct such surveys. The observations have always been difficult and the story is one of astronomers, goal-oriented in their quest to uncover the earliest population of galaxies, only making significant observational inroads when accompanying technological advances in telescope or instrument design take place. The first serious searches for PGs, carried out in the 1970s, utilised the large areal coverage obtainable with photographic plates (Partridge 1974), although it was only when optical charge-coupled device (CCD) technology was developed, in the early 1980s, that really useful limits were first reached.

\subsection{Optical continuum and emission-line searches}

A number of experiemnts have been carried out designed to detect the emission from redshifted Lyman $\alpha$-possibly the strongest single emission feature from young galaxies. One group of these observations utilises the technique of narrow-band imaging: a CCD image taken with a filter centred on redshifted Lyman $\alpha$ with a passband well-matched to the expected width of the emission line $(\simeq 20 \AA)$, greatly enhances the contrast of the line against the continuum of the galaxy and, more importantly, against the terrestrial sky-emission. In this way Pritchet and Hartwick (1987) set strong limits on the space density of PGs at $4.5<z<6$ from narrow-band imaging at wavelengths $6000 \leq \lambda \leq 8000 \AA$ using the Canada-France-Hawaii 3.6m telescope in Hawaii, while results on the same telescope by De Propris et al. (1993) using a system of narrow-band filters covering $4000 \leq \lambda \leq 5000 \AA$ found upper limits on Lyman $\alpha$ emission that are inconsistent with the simple model predictions discussed above by a factor of about 10 at redshifts $2 \leq z \leq 3$.

The other technique designed to detect redshifted Lyman $\alpha$ from PGs utilises a grating spectrograph incorporating a long object slit to maximise sky coverage during the observation. While the sky coverage obtainable is significantly less than that of the narrow-band technique, the advantages are that a significantly larger wavelength range can be observed simultaneously and the sky background can be subtracted accurately. For example, Thompson and Djorgovski (1995) used spectroscopic data from the 200 inch Hale telescope at the Palomar Observatory, obtained for other purposes over the course of a 7-year period, to search for serendipitous Lyman $\alpha$ emission over the wavelength range $5000 \leq \lambda \leq 7500 \AA$ and covering a total area of $15 \operatorname{arcmin}^{2}$ of sky (sufficient to contain at least 20 PGs) - placing limits on the SFR in PGs of $100 \mathrm{M}_{\odot} \mathrm{yr}^{-1}$.

Accompanying experimental limits on the brightness of the extragalactic background light at 
optical wavelengths are also consistent with the lack of success in detecting individual PGs at optical wavebands, helping to rule out the existence of a substantial PG population at $z<5$ (e.g. Dube, Wickes and Wilkinson 1977).

\subsection{The Era of the Near-Infrared}

Working at near-infrared wavelengths provides the opportunity to probe to even higher redshifts; the optical searches being limited to finding objects at $z \leq 5$. The earliest results in the near-infrared $(1-5 \mu \mathrm{m})$ were limited to experiments with single-element detectors: Boughn, Saulson and Uson (1986) placed some of the earliest limits at $2 \mu \mathrm{m}$ by searching for the fluctuations in the sky-brightness on scales $10-30$ arcsec - an experiment optimised to detect the signal of low surface brightness PGs at $z \sim 20$ overlapping on the sky. Collins and Joseph (1988) carried out what was probably the first near-infrared search for discrete PGs, looking for candidate objects with the expected flat spectral energy distribution (see figure 3). A summary of these early results is presented by Thompson, Djorgovski and Beckwith (1994), who conclude that with the limited field size and sensitivity available to near-infrared instruments at that time, the expected SFR of PGs could still be as high as $1000 \mathrm{M}_{\odot} \mathrm{yr}^{-1}$.

An important technological landmark for PG searches came with the advent of CCD-type array cameras for use in the $1-5 \mu \mathrm{m}$ waveband; in 1986 the 3.8m United Kingdom Infrared Telescope (UKIRT) in Hawaii was the first telescope to have such a general-purpose camera. In fact infrared array devices were constructed as early as 1974 but the technical information came late to astronomers due to the secrecy surrounding the early development which was largely carried out for military applications.

With these new panoramic detectors near-infrared searches began to rival their optical counterparts in limiting sensitivity and areal coverage: Parkes, Collins and Joseph (1994) placed the first really useful limits on the number density and Lyman $\alpha$ luminosity of objects at $7 \leq z \leq 9$ using narrow-band filters, while Pahre and Djorgovski (1995) achieved similar flux limits by targetting the emission lines of $\mathrm{H} \alpha,[\mathrm{OII}], \mathrm{H} \beta$ and $[\mathrm{O} \mathrm{II}]$ redshifted to $z=2.88$ and $z=4.79$ using the Keck 10m telescope situated alongside UKIRT in Hawaii. These limits constrain the

SFR of PGs to a value $\sim 1-10 \mathrm{M}_{\odot} \mathrm{yr}^{-1}$, similar to the constraints achieved in the optical.

One final avenue of investigation previously undertaken concerns radio searches for neutral hydrogen: From the lack of absorption lines at wavelengths shorter than Lyman $\alpha$ in the spectra of high redshift quasi stellar objects, it has been known for some time that the mass density in diffuse neutral hydrogen must be very small (Gunn and Peterson 1965). Nonetheless, a number of experiments have been carried out designed to detect redshifted $21 \mathrm{~cm}$ radiation from clumps of neutral hydrogen corresponding to an evolutionary stage of PGs preceeding the epoch at which stars form (Hogan and Rees 1979; Davies, Pedler and Mirabel 1978; Uson, Bagri and Cornwell 1991). A big drawback of these experiments as they currently stand is limiting sensitivity - the faintest of which corresponds to a neutral hydrogen mass $\sim 10^{14} \mathrm{M}_{\odot}$ at $z \geq 3$, which is much bigger than any predicted characteristic mass scale (see section 3). 


\subsection{Masquerading PGs}

Before looking at what a null detection of the starlight from PGs might mean it is worthwhile to consider if the problem lies in misunderstanding the nature of PGs and whether it is possible that PGs are masquerading as extragalactic objects of a type not yet considered: some galaxies exhibit poweful radio emission, resulting from a relativistic jet eminating from the core of the galaxy in which a black hole is believed to reside. This radio emission can be detected out to great distances and some of these objects are believed to be the site of large amounts star-formation: the source $3 \mathrm{C} 326.1$ at $z=1.8$ is forming stars at the rate of $300 \mathrm{M}_{\odot} \mathrm{yr}^{-1}$ (McCarthy et al. 1987); while the source called 4 C41.17 at $z=3.8$ emits $10^{13} \mathrm{~L}_{\odot}$ of starlight (where $1 \mathrm{~L}_{\odot} \simeq 3.8 \times 10^{26} \mathrm{~W}$ ) and contains enormous amonts of dust (Dunlop et al. 1994). Even though these objects are a powerful probe of the general characteristics of galaxies at high redshift, their strong radio signature isolates them as a special class of galaxy - too extreme to be the ancestors of more normal spirals and ellipticals.

Quasi stellar objects (QSOs) are at least candidates on the grounds that they occupy a similar redshift range to that expected of PGs - the furthest QSO has a redshift close to 5 - however, the comparison cannot be taken further than that; QSOs are powered by material accretion onto a black hole and their optical/near-infrared emission is dominated by the output from this process rather than starlight. What QSOs do tell us is that the high abundance of heavy elements, inferred from the absorption lines in their spectra, indicates significant chemical enrichment of the Universe along random lines-of-sight before $z \simeq 4$. The lack of a strong resemblance between other high-redshift objects such as QSOs and the expected characteristics of PGs forces us to conclude that PGs are not being mistaken for other extragalactic sources in large numbers and it is necessary to look for other reasons for their non-detection.

\section{Evidence for Type-Dependent Evolution of Galaxies}

\subsection{The Lyman $\alpha$ dropouts}

Although PG searches over the last 20 years have proved disappointing in uncovering a distant population of monolithic PGs, it is precisely this lack of success which has given rise in recent years to renewed interest in galaxy evolution at more modest redshift $(z \simeq 1-3)$, where galaxies are detected in numerous quantities by, for example, the Hubble Space Telescope.

These studies of evolution have proved pivital in our understanding the evolution of local galaxies, not by way of uncovering PGs as such - this is one discovery which has alluded the Hubble Telescope so far, but by measuring the rate at which stars are formed in normal spiral galaxies over a look-back time of a few Gyrs and have produced a rapid advance in studies of the Universe in the era of the 10m-class telescopes.

The basic technique used to find galaxies at these redshifts involves broad-band optical imaging in a number of filters to detect the sudden drop in the spectral energy distribution shortward of the redshifted Lyman break $(\leq 900(1+z) \AA)$. Photons with shorter wavelengths than this ionize hydrogen and are re-processed primarily into Lyman $\alpha$ photons. The resultant 


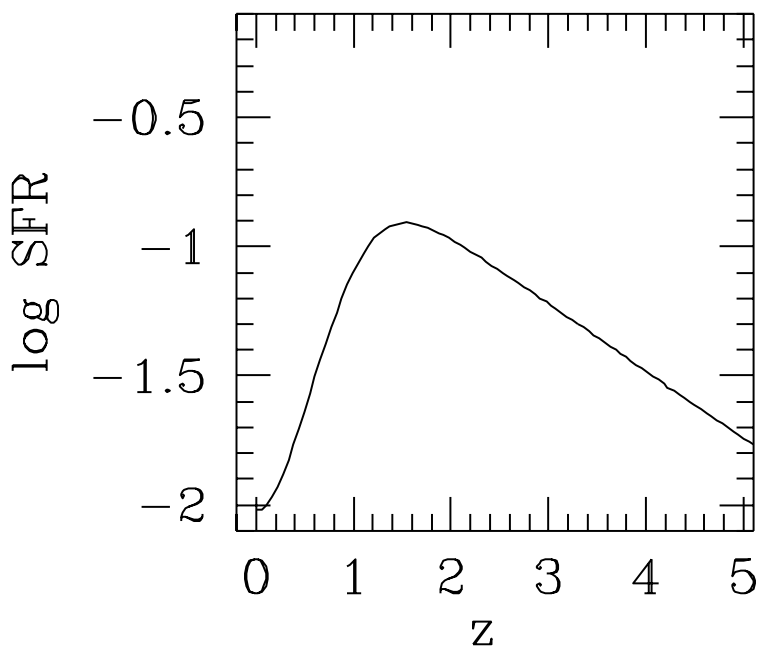

Figure 4: The implied star-formation rate $(\mathrm{SFR})$ in $\mathrm{M}_{\odot} \mathrm{yr}^{-1}$ against redshift inferred from several optically selected galaxy samples. The plot is taken from Madau et al. 1998.

characteristic spectral "step" can be seen in figure 3 for a zero redshift galaxy. In a series of experiments starting in 1992, Steidel and collaborators (see Steidel et al. 1996) have used this technique along with follow-up deep spectroscopy on the Keck telescope, to confirm the existence of a substantial population of compact $(\sim 0.4$ arcsec $)$ star-forming galaxies at $z \sim 3$. From the strength of their emission lines and shape of their continua, these galaxies have SFRs $\leq 30 \mathrm{M}_{\odot} \mathrm{yr}^{-1}$ and a number density equivalent to between $50 \%-10 \%$ of the space density of present day bright galaxies. Progress in this area has been very rapid and it is now possible for the first time to sketch out the star-forming history of a substantial fraction of the present day galaxy population (Madau et al. 1996): The work of Steidel, together with other samples selected in a similar manner - including galaxies found by the Hubble Space Telescope (e.g. Lilly et al. 1996, Connolly et al. 1997), indicate that the overall SFR of galaxies increases from $z=0$ to $z=2$, during which time a significant fraction of the heavy elements in the Universe are formed, and then tails off towards higher redshift - see figure 4 taken from Madau et al. (1998). Although the uncertainties are considerable and many surveys are still underway, figure 4 is a real landmark in cosmology as it represents the very first attempt to map out the star-forming history of galaxies.

The Lyman dropout galaxies are known to be young from the shape of their spectral energy distributions, which are quite flat (see figure 3), and they also show signs of energetic outflows consistent with intense bursts of star formation (Pettini et al. 1998). So in key respects this widespread population of objects at $z \sim 3$ has some of the essential characteristics which we 
expect from PGs and it is quite possible that we are seeing directly, for the first time, the formation of present day galaxies. These results certainly demonstrate beyond any reasonable doubt that massive galaxy formation was well underway by $z=3.5$. This relatively late epoch of galaxy formation appears to be in general agreement with the popular models of galaxy formation in which the mass density of the Universe is dominated by cold dark matter, discussed in section 3, which predict a peak SFR of a few $\mathrm{M}_{\odot} \mathrm{yr}^{-1}$ between $2 \leq z \leq 4$ (Baugh et al. 1998).

\subsection{The Age of Elliptical Galaxies}

Despite the discovery of the Lyman dropout population at moderate redshift, the alternative view that galaxies formed at high redshift in a short but intense period of star formation still provides the best explanation for the formation of at least some galaxies - in particular, elliptical galaxies. These constitute $\sim 30 \%$ of the galaxy population seen at low redshift and ellipticals seen at $z \simeq 0.5$ have spectral energy distributions conststent with a passively evolving stellar population with an age of several Gyrs (see figure 3), equivalent to a formation redshift $z \geq 5$ (e.g. Bender et al. 1996, Ellis et al. 1997). So a complete picture of when galaxies form remains elusive: nearby elliptical galaxies, the most massive of any galaxy-type, contain a stellar population too old for them to be the low redshift counterparts of the Lyman dropout galaxies and yet primeval elliptical galaxies are not seen in the deep optical or near-infrared searches for monolithic PGs. A key question then is: how can the dearth of PGs in the optical and near-infrared searches (described in section 5), many of which are tuned to detect objects at $z \simeq 5$, be reconciled with the seemingly inevitable conclusion from evolution studies that this is exactly the epoch at which elliptical galaxies are expected to form?

\section{Dusty Elliptical PGs}

By far the most likely answer to this question is that young elliptical galaxies are enshrouded in the dust formed by young stars, which absorbs the starlight, making them almost invisible at optical and near-infrared wavelengths. Figure 5 shows the effect of only a small amount of dust on the optical and UV light eminating from a young galaxy 1 Gyr old. The absorbed energy simply gets re-radiated as thermal emission giving rise to substantial emission at far-infrared and sub-millimetre wavelengths.

In a parallel way to that of near-infrared astronomy some 10 years ago, recent technological developments in the last couple of years have led to the first searches for dusty PGs: SCUBA (the Submillimetre Common User Bolometer Array) was built by the Royal Observatory Edinburgh for use on the James Clerk Maxwell Telescope (JCMT) in Hawaii. It is the world's largest submillimetre camera, and takes images simultaneously at two submillimetre wavelengths. It can take deeper images of the sub-millimetre $(450 \mu \mathrm{m}-2.0 \mathrm{~mm})$ sky than ever before, map-

ping areas in minutes which previously used to take hours to complete and is quite capable of detecting re-processed starlight from dusty PGs at high redshift.

Fueling the excitment surrounding the anticipated arrival of SCUBA was the report of the 


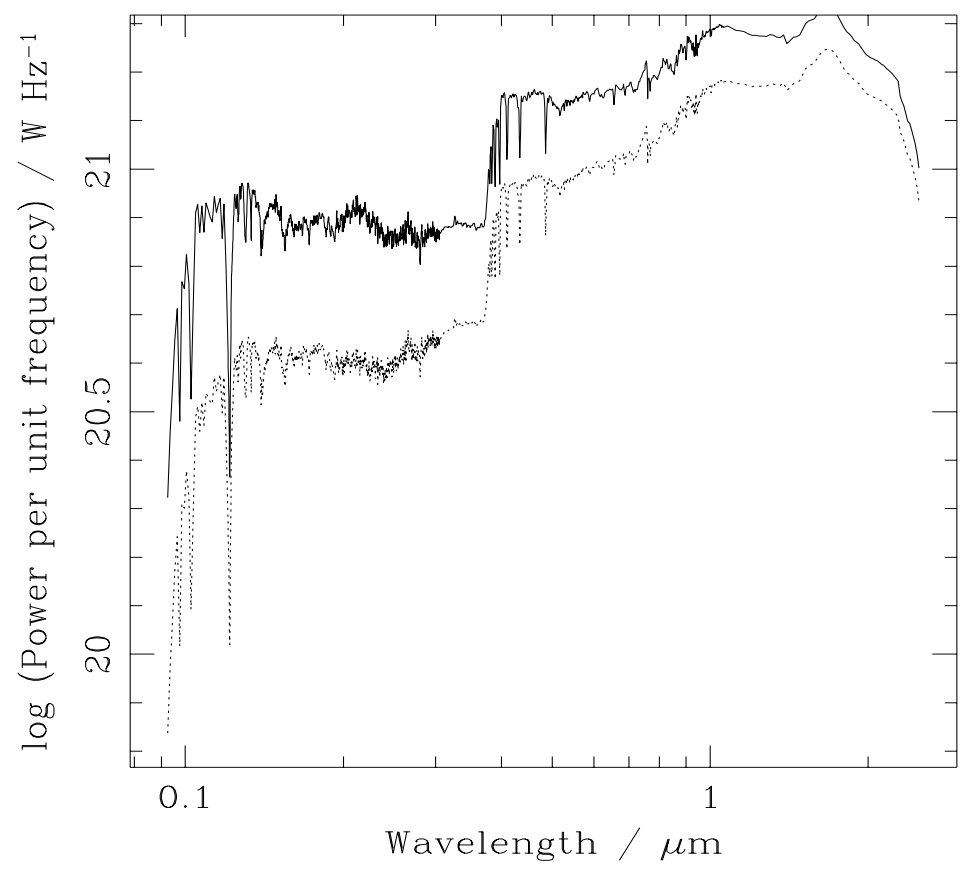

Figure 5: The spectral energy distribution of a galaxy 1 Gyr old forming stars at a rate of $1 \mathrm{M}_{\odot} \mathrm{yr}^{-1}$. The top curve is the prediction with no dust (as in figure 3 ) and the bottom curve the prediction assuming a simple dust creation model.

first detection of an extragalactic far-infrared background from the COBE satellite (Puget et al. 1996). This background appears isotropic over the range $400 \mu \mathrm{m}-1.0 \mathrm{~mm}$ and has a natural explanation in terms of re-radiated starlight from dusty galaxies at high redshift (see Puget et al. 1996). Despite only begining regular observations in May 1997, SCUBA has not proved disappointing: detections of sources with submillimetre spectral properties consistent with high redshift dusty PGs were reported last year (Smail et al. 1997). Some caution is necessary in the early interpretion of these results since the Smail et al search fields cover only a few $\operatorname{arcmin}^{2}$ of sky and were not the traditional blank fields characteristic of previous PG searches, but instead were centered on known distant clusters of galaxies. This was done in order to utilise the familiar gravitational lensing effect seen in rich clusters, which amplifies the light from backgound objects as it passes through the cluster's gravitational potential well. While this makes distant galaxies brighter and therefore easier to detect, source luminosities and surface density estimates require accurate knowledge of the amplification factor, which is intrinsically uncertain.

Very recently, the results from two independent blank field searches have been reported (Hughes et al. 1998, Barger et al. 1998). Each group surveyed approximately $10 \operatorname{arcmin}^{2}$ of sky (including the celebrated Hubble Deep Field). These data reveal objects with submillimetre spectral properties consistent with that expected from dusty galaxies in the redshift range $z=2-4$, powered by star formation with an implied SFR 5-10 times larger than the Lyman 
dropout galaxies seen in optical surveys. Results from surveys covering larger areas of sky are eagerly awaited: if these and similar experiments confirm a dusty PG population, the last empirical ingredient required to understand the formation of galaxies may have been found and a description of galaxy evolution over a wide span of cosmic time would then be within reach.

\section{Concluding Remarks and Future Prospects}

This is a very rapidly changing field of research. The advent of the 10m-class optical telescopes such as Keck, soon to be joined by others, has revolutionised our understanding of galaxy evolution in recent years and much still can be done in the optical and near-infrared: the recent discoveries of Lyman $\alpha$ emission from a few galaxies at $z=3-6$ (Dey et al. 1998, Hu et al. 1998), shows that not all galaxies at this epoch are dusty, which provides fresh hope for the discovery of PGs when the new generation of even larger near-IR arrays becomes available on the largest telescopes. Certainly the US space agency NASA recognises the importance of continuing PG searches: over the next two decades it has dedicated itself to the "cosmic origins" programme, designed to answer such questions as "how did the first galaxies form?" This will require a succession of sophisticated telescopes, each building on the results of previous missions augmented with ground-based observations. One such project is the New Generation Space Telescope - a $4 \mathrm{~m}$ (or possibly $8 \mathrm{~m}$ ) descendant of the incumbent Hubble Space Telescope, designed for diffraction-limited imaging over $1 \mathrm{deg}^{2}$. With such capability it will be possible to observe the individual supernovae explosions resulting from massive bursts of star formation happening at almost any epoch.

Undoubtedly, far-infrared and submillimetre observations will have future rôle to play in the unfolding story: the Planck satellite mission (named after the German physicist Max Planck), is part of the European Space Agency's Horizon 2000 Scientific Programme. Due to be launched in $\simeq 2005$, Planck will survey the whole sky at millimetre wavelengths with unprecedented sensitivity and angular resolution. Designed primarilly to map fluctuations in the cosmic microwave background, Planck has the capability to detect small fluctuations in the far-infrared background tentatively detected by COBE, thereby distinguishing between rival theories predicting different epochs of galaxy formation. Perhaps the most exciting prospect on the timescale of $10 \mathrm{yrs}$ or so, is the development of millimetre array imagers, capable of both high spatial and spectral resolution imaging over the whole sub-millimetre waveband. For example, the National Radio Astronomical Observatories millimetre array, proposed to the US National Science Foundation will provide a spatial resolution of 10 milliarcsec between $10 \mathrm{~mm}-350 \mu \mathrm{m}$ and a sensitivity capable of detecting the dust emission from a bright star-forming galaxy with luminosity $>10^{11} \mathrm{~L}_{\odot}$ to $z=20$. 


\section{Acknowledgements}

I would like to thank Gordon Rogers for the spectral energy distribution plots and Doug Burke for useful discussions.

\section{References}

Barger, A.J., Cowie, L.L., Sanders, D.B., Fulton, E., Taniguchi, Y., Sato, Y., Kawara, K., and Okuda, H., 1998, Nature, 394, 248.

Baugh, C.M., Cole, S., Frenk, C.S., and Lacey, C.G., 1998, Astrophysical Journal, 498, 504.

Bender, R., Ziegler, B., Bruzual, G., 1996, Astrophysical Journal, 463, L51.

Boughn, S.P., Saulson, P.R., and Uson, J.M., 1986, Astrophysical Journal, 301, 17.

Charlot, S., Worthey, G., Bressan, A., 1996, Astrophysical Journal, 457, 625.

Collins, C.A., and Joseph, R.D., 1988, Mon. Not. R. astr. Soc., 235, 209.

Connolly, A.J., Szalay, A.S., Dickinson, M.E., Subbarao, M.U., and Brunner, R.J., 1997, Astrophysical Journal, 486, 11.

Davies, R.D., Pedlar, A., and Mirabel, I.F., 1978, Mon. Not. R. astr. Soc., 182, 727.

De Propris, R., Pritchet, C.J., Hartwick, F.D.A., and Hickson, P., 1993, Astronomical Journal, 105, 4.

Dey, A., Spinrad, H., Stern, D., Graham, J.R., and Chaffee, H., 1998, Astrophysical Journal, 498, L93.

Dube, R., Wickes, W.C., and Wilkinson, D.T., 1977, Astrophysical Journal, 215, L51.

Dunlop, J.S., Hughes, D.H., Rawlings, S., Eales, S., and Ward, M.J., 1994, Nature, 370, 347.

Eggen, O.J., Lynden-Bell, D., and Sandage, A.R., 1962, Astrophysical Journal, 136, 748.

Ellis, R.S., 1997, Ann. Rev. Astron. Astrophys., 1997, 35, 389.

Ellis, R.S., Smail, I., Dressler, A., Couch, W.J., Oemler, A., Butcher, H., and Sharples, R.M., 1997, Astrophysical Journal, 483, 582.

Gunn, J.E., and Peterson, B.A., 1965, Astrophysical Journal, 142, 1633.

Hogan, C.J., and Rees, M.J., 1979, Mon. Not. R. astr. Soc., 188, 791.

Hughes, D., Serjeant, S., Dunlop, J., Rowan-Robinson, M., Blain, A., Mann, R.G., Ivison, R., Peacock, J., Efstathiou, A., Gear, W., Oliver, S., Lawrence, A., Longair, M., Goldschmidt, P., and Jenness, T., 1998, Nature, 394, 241.

Hu, E., Cowie, L., and McMahon, R., 1998, Astrophysical Journal, 502, L99.

Kennicutt, R.C., Keel, W.C., van der Hulst, J.M., Hummel, E., Roettiger, K.A., 1987, Astronomical Journal, 93, 1011.

Koo, D., and Kron, R., 1992, Annu. Rev. Astron. Astrophys., 30, 613.

Larson, R.B., 1974, Mon. Not. R. astr. Soc., 145, 405.

Lilly, S.J., Le Févre, O., Hammer, F., and Crampton, D., 1996, Astrophysical Journal, 455, 108.

Madau, P., Ferguson, H.C., Dickinson, M.E., Giavalisco, M., Steidel, C.C., Frchter, A., 1996, Mon. Not. R. astr. Soc., 283, 1388.

Madau, P., Pozzetti, L., and Dickinson, M., 1998, Astrophysical Journal, 498, 106.

McCarthy, P., Spinrad, H., Djorgovski, S., Strauss, M. A., van Breugel, W., and Leibert, J., 1987, Astrophysical Journal, 319, L39.

Pahre, M.A., and Djorgovski, S.G., 1995, Astrophysical Journal, 449, L1.

Parkes, I. M., Collins, C.A., and Joseph, R.D., 1994, Mon. Not. R. astr. Soc., 266, 983. 
Partridge, R.B., 1974, Astrophysical Journal, 192. 241.

Partridge, R.B., and Peebles, P.J.E., 1967, Astrophysical Journal, 147, 868.

Penzias, A.A., and Wilson, R.W., 1965, Atrophysical Journal, 142, 419.

Pettini, M., Steidel, C.C., Adelberger, K., Kellogg, M., Dickinson, M., and Giavalisco, M., 1998, In Cosmic Origins: Evolution of galaxies, stars, planets and life, eds. Woodward, C.E., and Thronson, H.A., (Astronomical Society of the Pacific Conference Series), in press.

Pritchet, C.J., 1994, Publications of the Astronomical Society of the Pacific, 106, 1052.

Pritchet, C.J., and Hartwick, F.D.A., 1987, Astrophysical Journal, 320, 464.

Puget, J.L., Abergel, A., Bernard, J.P., Boulanger, F., Burton, W.B., Désert, F.X., and Hartmann, D., 1996, Astronomy and Astrophysics, 308, L5.

Sandage, A.R., 1995, In The Deep Universe, eds. Binggeli, B., Buser, R., (Springer: Berlin), 1.

Smail, I., Ivison, R.J., and Blain, A.W., 1997, Astrophysical Journal, 490, L5.

Smoot, G., and Keay, D., 1993, Wrinkles in time, (William Morrow: New York).

Steidel, C.C., Giavalisco, M., Pettini, M., Dickinson, M., and Adelberger, K.L., 1996, Astrophysical Journal, 462, 17.

Thompson, D., and Djorgovski, S., 1995, Astronomical Journal, 110, 3.

Thompson, D., Djorgovski, S., and Beckwith, S.V.W., 1994, Astronomical Journal, 107, 1.

Uson, J.M., Bagri, D.S., and Cornwell, T.J., 1991, Astrophysical Journal, 377, L65. 


\section{Biographical Details}

Dr Collins carried out his PhD work at Imperial College, London University, under the supervision of Dr R. Joseph from 1982-1985. His thesis work was based largely on an infrared search for primeval galaxies. Since then he has been a research fellow at the University of Edinburgh and a senior research fellow at the Royal Observatory Edinburgh. In 1992 he moved to Durham University on a PPARC Advanced Fellowship and in 1994 became a lecturer at Liverpool John Moores University. He was promoted to Professor in 'Cosmology' in September 1998 . His other research interests are large-scale structure in the Universe, the evolution of galaxies, and the evolution of X-ray emitting galaxy clusters. 